\title{
Treponema maltophilum sp. nov., a Small Oral Spirochete Isolated from Human Periodontal Lesions
}

\author{
C. WYSS, ${ }^{1 *}$ B. K. CHOI, ${ }^{2}$ P. SCHÜPBACH,${ }^{1}$ B. GUGGENHEIM, ${ }^{1}$ AND U. B. GÖBEL ${ }^{2}$ \\ Institute for Oral Microbiology and General Immunology, Center for Dentistry, Oral Medicine and Maxillofacial \\ Surgery of the University of Zürich, CH-8028 Zürich, Switzerland, ${ }^{1}$ and Universitätsklinikum \\ Charité, Institut für Mikrobiologie und Hygiene, D-10117 Berlin, Germany ${ }^{2}$
}

\begin{abstract}
A novel culture medium for cultivation of fastidious oral anaerobes is described. This medium, OMIZ-Pat, consists of a rich chemically defined basal medium supplemented with asialofetuin, as well as yeast extract and Neopeptone fractions. Addition of $1 \mathrm{mg}$ of rifampin per liter and $100 \mathrm{mg}$ of fosfomycin per liter allowed routine isolation of spirochetes by a limit dilution method in 96-well plates containing liquid OMIZ-Pat. In addition to members of the four previously recognized species of oral treponemes (Treponema denticola, Treponema pectinovorum, Treponema socranskii, and Treponema vincentii), 26 previously undescribed spirochete strains belonging to one group were isolated. We propose the name Treponema maltophilum sp. nov. for these small spirochetes, which have two endoflagella; one endoflagellum is attached at each cell pole, and the endoflagella overlap in the middle of the cell. Growth of these organisms was dependent on a carbohydrate like D-arabinose, L-fucose, D-maltose, L-rhamnose, D-ribose, D-sucrose, or D-trehalose and was inhibited by fetal bovine serum. T. maltophilum is distinguished from other oral Treponema species by its 16S rRNA sequence, its protein and antigen patterns as determined by sodium dodecyl sulfate-polyacrylamide gel electrophoresis and immunoblotting, and its characteristic $\alpha$-glucosidase activity. The strains included in the new species on the basis of their 16S rRNA sequences are heterogeneous with respect to their $\alpha$-fucosidase and $\beta$-glucuronidase activities, their dependence on $\mathrm{N}$-acetylglucosamine, and their antigens as detected with patient antibodies. Strain BR is designated the type strain, and strains HO2A and PNA1 are reference strains of the new species.
\end{abstract}

Investigators of the etiology of periodontal diseases have documented the enormous complexity of the microbial populations of dental plaque (22). Hundreds of species have been isolated and identified by cultural techniques. However, it is widely accepted that numerous organisms present in plaque remain entirely unnoticed or are recognized microscopically because of their prominent morphology but have never been isolated. This could explain the failure so far to identify bona fide periodontopathogens $(11,24)$.

Recently, we established a library of 16S rRNA sequences from the bacteria present in a single sample of subgingival plaque from a periodontitis patient. This library defined in a culture-independent way the spectrum of genotypes that were most prevalent in that population (6). When the spirochetes were considered, this spectrum proved to be far wider than the spectrum of oral treponemes that have been cultured. Although this approach yielded no information concerning the physiological characteristics of these organisms, it opened the way for developing rapid analytical tools to specifically detect these genotypes in plaque samples.

Improved in vitro culture techniques have been developed to investigate the physiology of established laboratory strains of oral anaerobes under defined conditions, in particular the metabolic activities important for plaque ecology, and to detect possible virulence factors $(36,37,40)$. It was expected that refinements in these culture techniques would expand the spectrum of fastidious anaerobes amenable to in vitro analysis.

In this paper we describe a novel culture technique that allowed isolation and characterization of previously uncultured oral treponemes. Although phenotypically heterogeneous, these organisms fell into a single phylotype that previously was iden-

* Corresponding author. Mailing address: Institute for Oral Microbiology and General Immunology, Plattenstr. 11, CH-8028 Zürich, Switzerland. Phone: 01/2573322. Fax: 01/2615683. tified on the basis of 16S rRNA sequence criteria (6), for which we propose the name Treponema maltophilum.

\section{MATERIALS AND METHODS}

Bacteria. The following type and reference strains were obtained and maintained as described previously (37): Treponema denticola CD-1, 51B2, ATCC 33521 , ATCC 35404 , and ATCC $35405^{\mathrm{T}}$ ( $\mathrm{T}=$ type strain); Treponema pectinovorum ATCC $33768^{\mathrm{T}}$; Treponema socranskii subsp. buccale ATCC $35534^{\mathrm{T}}$; Treponema socranskii subsp. paredis ATCC $35535^{\mathrm{T}}$; Treponema socranskii subsp. socranskii ATCC $35536^{\mathrm{T}}$; Treponema vincentii LA-1 (= ATCC 35580) and Ritz A; and Treponema sp. strain ATCC 43242.

Culture media. To enhance the recovery of organisms that may be more fastidious than the laboratory-adapted reference strains, a number of supplements were added to chemically defined liquid medium OMIZ-W1 (37). Initially, we used an enriched medium, OMIZ-WP, which was prepared from OMIZ-W1 by adding glutathione, asialofetuin (AsF), a methanol-soluble fraction of yeast extract (YEM), and a deanionized fraction of Difco Neopeptone (DANP) (39). After the first results for carbohydrate utilization by strain $\mathrm{BR}^{\mathrm{T}}$ were obtained, $2 \mathrm{~g}$ of D-mannose per liter, $2 \mathrm{~g}$ of $\mathrm{D}$-arabinose per liter, $2 \mathrm{~g}$ of $\mathrm{L}$-fucose per liter, $2 \mathrm{~g}$ of $\mathrm{D}$-trehalose per liter, $2 \mathrm{~g}$ of $\mathrm{D}$-sucrose per liter, and $2 \mathrm{~g}$ of $\mathrm{L}$-rhamnose per liter were added, and the resulting medium was designated OMIZ-Pat. All comparative studies were performed with cells grown in OMIZ-Pat. The solid media used for colony cloning were prepared as described previously for semisolid media (37), except that the final concentration of agarose (catalog no. 05068; Fluka, Buchs, Switzerland) was increased from 0.25 to $1.5 \%$.

Limit dilution culture analysis of plaque bacterial populations. All plaque samples were obtained from patients with recurrent, treatment-resistant periodontitis. Subgingival plaque samples were collected from dental pockets that were $>5 \mathrm{~mm}$ deep by using paper points and were transferred into reduced transport medium (17). The bacteria were released and dispersed by vortexing. Samples were processed within $3 \mathrm{~h}$ of collection. All procedures were performed in ambient air without prereduction of media. Samples were diluted with OMIZPat to determine the total counts and with OMIZ-Pat supplemented with antibiotics to select for spirochetes. A dilution of $10^{-7}$ for nonselective medium and a dilution of $10^{-5}$ to $10^{-6}$ for the antibiotic-containing medium usually resulted in growth in about $50 \%$ of the wells. The wells in one 96-well plate were filled with $0.2 \mathrm{ml}$ of medium per well for each dilution of plaque samples. After 10 days of incubation at $36^{\circ} \mathrm{C}$ in GasPak anaerobic jars (BBL), the wells were examined to determine growth and cell morphology by using an inverted phase-contrast microscope (Olympus). In our current procedure for selective isolation of oral spirochetes we use OMIZ-Pat supplemented with $1 \mathrm{mg}$ of rifampin per liter and $100 \mathrm{mg}$ of fosfomycin per liter. Samples from wells containing spirochetes were streaked onto OMIZ-Pat agarose plates, and within 5 to 10 days spirochetes 
formed diffuse colonies entirely within the agarose. Colonies were punch collected with glass Pasteur pipettes and transferred into liquid OMIZ-Pat. Clones were incubated until visible turbidity developed and stored in liquid nitrogen after $10 \%$ glycerol was added to the growth medium. Cultures were restreaked onto agarose to confirm homogeneity and then characterized.

Electron microscopy. To visualize endoflagella, cells were suspended for $5 \mathrm{~min}$ in a $0.2 \%$ aqueous solution of the nonionic detergent Teepol $610 \mathrm{~S}$ (catalog no. 86350; Fluka), to remove the outer cell membrane. After negative staining with $2 \%$ phosphotungstic acid (2), the cells were examined with a Philips model 201 transmission electron microscope. For scanning electron microscopy with a Cambridge Stereoscan S-180 microscope, whole cells were allowed to attach to cationized coverslips, fixed for $2 \mathrm{~h}$ in $2 \%$ glutaraldehyde buffered at $\mathrm{pH} 7.4$ with 0.1 NI sodium cacodylate, dehydrated by using increasing concentrations of acetone, dried in a critical point apparatus (model CPD-030; Bal-Tec, Balzers, Liechtenstein), and sputter coated with gold (model MED-010; Bal-Tec).

Effects of medium components on growth. Cells were grown in OMIZ-Pat, and samples were diluted at least 1:1,000 with semisolid test medium and dispensed into 24-well plates (Nunc, Roskilde, Denmark) in which duplicate wells had been preloaded with test compounds. Incubation and evaluation were performed as described previously $(37,38)$. The test medium used to assay for the effects of different sugars on growth and acid production was OMIZ-WP prepared without the carbohydrates present in OMIZ-W1 (glucose, fructose, maltose, citric acid, mannitol, glucuronic acid, and galacturonic acid). The following sugars were tested individually at a final concentration of $2 \mathrm{mg} / \mathrm{ml}$ : D-arabinose, $\mathrm{D}$-cellobiose, $\mathrm{D}$-fructose, D-fucose, D-galactose, D-galacturonic acid, D-glucose, D-glucuronic acid, D-lactose, D-maltose, D-mannitol, D-mannose, D-melibiose, D-ribose, D-sucrose, D-trehalose, D-xylose, L-arabinose, L-fucose, L-rhamnose, L-sorbose, and L-xylose. The test medium used to study the effects of $N$-acetylglucosamine (GlucNac), AsF, YEM, and DANP on the growth of treponemes was OMIZ-Pat without these four components. The test medium used to study the effects of antibiotics and fetal bovine serum (FBS) (Gibco BRL) was OMIZ-Pat.

Enzyme activities. Reference strains and new isolates were grown in OMIZ Pat and centrifuged. To test for catalase activity, cell pellets were covered with $3 \% \mathrm{H}_{2} \mathrm{O}_{2}$ and observed for $15 \mathrm{~min}$ for bubble evolution. For API ZYM tests the cells were resuspended in $0.9 \% \mathrm{NaCl}$ and examined as recommended by the supplier (Bio Mérieux SA, Marcy-l'Etoile, France)

Sodium dodecyl sulfate (SDS) gels and Western blots (immunoblots). For protein and antigen pattern analyses all strains were grown in OMIZ-Pat and processed for polyacrylamide gel electrophoresis (PAGE). Extracts were electrophoresed on gels with a 7.5 to $15 \%$ acrylamide gradient, electroblotted onto nitrocellulose paper, and immunolabelled as described previously (35). Before immunolabelling, the blots were stained and destained for protein (1). Patient sera were used at a dilution of 1:50, and an alkaline phosphatase-labelled second reagent was used at a dilution of 1:1,000; naphthol AS-E phosphate was used as the substrate for the phosphatase (33).

DNA isolation. Genomic DNA was isolated from 10-ml treponeme cultures in OMIZ-Pat as described previously (6), with the following modifications: (i) incubation with lysozyme was omitted; (ii) incubation with proteinase $\mathrm{K}$ was performed for $2 \mathrm{~h}$ at $37^{\circ} \mathrm{C}$; and (iii) after washing with $70 \%$ ethanol, the DNA was dissolved in $50 \mu \mathrm{I}$ of TE buffer (10 mM Tris- $\mathrm{HCl}$ [pH 8.0], 1 mM EDTA) and stored at $-20^{\circ} \mathrm{C}$.

16 S ribosomal DNA sequencing. 16S rRNA sequences were amplified by PCR by using universal primers TPU1 (5'AGAGTTTGATC/A/C]TGGCTCAG3'; positions 8 to 27 in Escherichia coli 16S rRNA) and RTU8 (5'AAGGAGGT GATCCA[T/G]CC[G/A]CA3'; positions 1541 to 1522 in E. coli $16 \mathrm{~S}$ rRNA) as described previously (6). Amplicons were purified by filtration by using Centricon 100 membrane filters (Amicon, Witten, Germany) and were sequenced by a modified Sanger dideoxynucleotide chain termination method, using a Sequenase sequencing kit (Amersham, Amersham, Buckinghamshire, United Kingdom) and universal eubacterial primers $(14,32)$ as described by Casanova et al. (3). The $16 \mathrm{~S}$ ribosomal DNA sequences obtained were compared with all currently available sequences in public (EMBL, GenBank) and private $(5,7)$ databases by using the sequence analysis program Husar 4.0 (Deutscher Krebsforschung Zentrum, Heidelberg, Germany). To construct phylogenetic trees, we used the TREECON version 3.0 software package (31). Because only a partial sequence of $T$. vincentii was available, we constructed a distance matrix based on about 900 bases ( $E$. coli positions 54 to 930 ), and multiple base changes at single positions were corrected by the method of Jukes and Cantor (13). A phylogenetic tree was constructed by using the neighbor-joining method of Saitou and Nei (27).

Nucleotide sequence accession numbers. The EMBL accession numbers for the reference spirochetal 16S rRNA sequences which we used are as follows: Treponema succinifaciens, M57738; Treponema sp. strain CA, M59294; Treponema saccharophilum, M71238; Treponema bryantii, M57737; T. pectinovorum, M71237; Treponema pallidum, M88726; Treponema phagedenis, M57739; T. denticola, M71236; Spirochaeta sp., M71240; Spirochaeta isovalerica, M88720; Spirochaeta aurantia, M57740; Borrelia burgdorferi, L36160; Borrelia hermsii, M60968; Borrelia anserina, M72397; Brachyspira aalborgi, Z22781; Serpulina innocens, M57744; Serpulina hyodysenteriae, M57742; Leptonema illini, Z21632; Leptospira interrogans, X17547; and Leptospira biflexa Z12821. The 16S ribosomal DNA sequence of strain $\mathrm{BR}^{\mathrm{T}}$ determined in this study is available from the EMBL,
GenBank, and DDBJ nucleotide sequence databases under accession number X87140.

\section{RESULTS}

Using a limit dilution technique and various modifications of the chemically defined culture medium OMIZ-W1 (37), we isolated a group of small, saccharolytic, serum-sensitive spirochetes which, on the basis of various criteria, were distinct from previously cultured oral Treponema species. A total of 26 clones of these novel organisms were obtained from 13 subgingival plaque samples from nine patients with periodontitis. When they were streaked onto OMIZ-Pat agarose plates, the new isolates formed within 5 days off-white, diffuse colonies that were up to $3 \mathrm{~mm}$ in diameter and completely penetrated the agarose. As shown in Fig. 1 for strain $\mathrm{BR}^{\mathrm{T}}$, these small spirochetes (diameter, $0.2 \mu \mathrm{m}$; wavelength, $0.7 \mu \mathrm{m}$; amplitude, $0.3 \mu \mathrm{m}$ ) had two endoflagella, one attached subterminally at each pole. More than 50 cells of each of the 11 new strains included in Fig. 2 were analyzed; in all but some very long cells the endoflagella were long enough to overlap in the central region of the periplasmatic space (i.e., the flagellation type was type 1:2:1). The cell length usually corresponded to six to eight wavelengths, but could be quite variable, as is often the case for spirochetes in culture (34). In liquid media the cells did not exhibit much twitching movement but rather rotated around their axes, often producing waves with estimated amplitudes of up to $2 \mu \mathrm{m}$ but not achieving translational movement. However, in viscous medium (soft agarose) or when bacteria attached to the surface of a glass coverslip, cellular rotation resulted in serpentine translation; the direction of movement was changed by reversing the rotation and/or bending the cell body.

Protein and antigen pattern. A comparison of the protein and antigen patterns of cultured oral treponemes after separation by SDS-PAGE and transfer to nitrocellulose paper (blots) is shown in Fig. 2. The new isolates exhibited considerable heterogeneity, but were readily grouped together and were clearly distinguishable from the reference strains of $T$. denticola, $T$. pectinovorum, $T$. socranskii subsp. buccale, $T$. socranskii subsp. paredis, $T$. socranskii subsp. socranskii, and T. vincentii (Fig. 2A). Sera from periodontitis patients were used as polyvalent immunostaining reagents. The resulting antigen patterns on the blots (Fig. 2B through D) provided further detail concerning the heterogeneity of the new isolates and their differentiation from other species. In addition to detecting apparently species-specific antigens (Fig. 2B and D) and numerous strain-specific antigens (Fig. 2B through D), immunostaining with some sera revealed a ca. $75-\mathrm{kDa}$ antigen only in strains that exhibited $\alpha$-fucosidase activity (Fig. 2C, lanes 6 through 11; same blot as the blot in Fig. 2A immunostained with serum from the patient harboring strain $\mathrm{BR}^{\mathrm{T}}$ ). Figure 2D shows the results of immunolabelling with serum from the patient who harbored the strains in lanes 5,7 , and 8 , which exhibited marked reactivity with a ca. $100-\mathrm{kDa}$ antigen in all of the new isolates except two (the strains in lanes 7 and 8 ), which may have been selected by this immune response. A third strain from the same patient which did exhibit this antigen activity (lane 5) was isolated from a different plaque sample.

Nutrient requirements. As shown in Table 1, strains $\mathrm{BR}^{\mathrm{T}}$, $\mathrm{HO} 2 \mathrm{~A}$, and PNA1 differed markedly in their requirements for YEM, DANP, AsF, and GlucNac. GlucNac was essential for growth of all of the novel isolates with $\alpha$-fucosidase activity tested, while it was highly stimulatory but not necessary for growth of the strains that lacked $\alpha$-fucosidase activity. In con- 

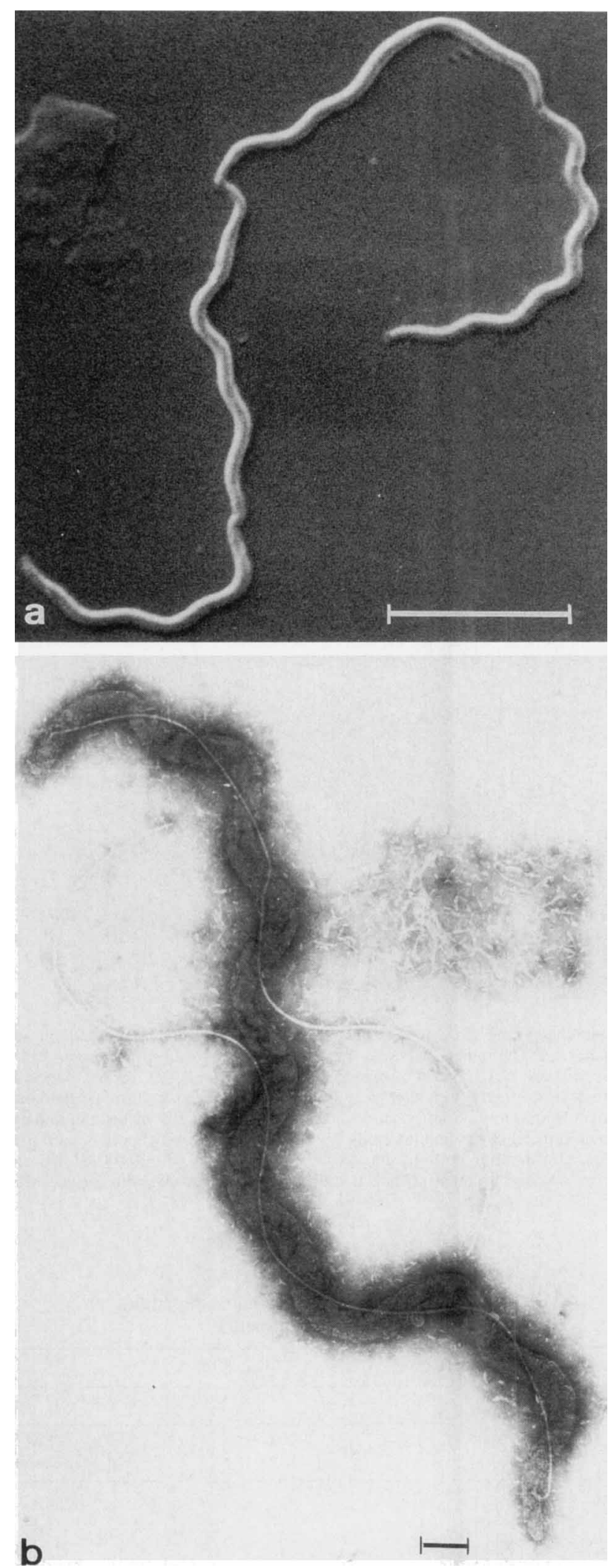

FIG. 1. (a) Scanning electron micrograph of strain $B^{T}$ cells. Bar $=2 \mu \mathrm{m}$. (b) Transmission electron micrograph of a strain $\mathrm{BR}^{\mathrm{T}}$ cell with the outer cell membrane removed. Note the two endoflagella, one attached subterminally at each pole. These flagella are long enough to overlap in the central region of cell body. The preparation was negatively stained. Bar $=2 \mu \mathrm{m}$. trast, the growth of laboratory strains and clinical isolates of the other four species of cultivable oral spirochetes is not influenced by GlucNac (data not shown).

Addition of 5\% FBS to OMIZ-Pat eliminated the growth of all of the new isolates. Consistent with the results of our previous studies performed with chemically defined medium OMIZ-W1 (37), addition of FBS to OMIZ-Pat inhibited the growth of all $T$. socranskii strains, whereas the growth of $T$. denticola, $T$. pectinovorum, and $T$. vincentii either was not affected or was enhanced by FBS (data not shown).

The effects of individual carbohydrates on the growth of oral treponemes in OMIZ-Pat containing GlucNac but no other additional carbohydrates are summarized in Table 2 . All but three isolates responded to maltose, although most strains, including strains $\mathrm{BR}^{\mathrm{T}}$ and $\mathrm{HO} 2 \mathrm{~A}$, were not able to utilize glucose. The new isolates that were not stimulated by maltose (including PNA1) grew well in the absence of any carbohydrate. Acid production as detected by a change in the color of phenol red from red to yellow was observed for all of the new isolates, as well as all of the $T$. socranskii and $T$. pectinovorum strains tested; no $T$. denticola or $T$. vincentii strain produced acid in response to sugar addition, although the growth of some strains was enhanced. The two new strains that grew on glucuronic acid (including strain $\mathrm{HO} 2 \mathrm{~A}$ ) were the strains that exhibited $\beta$-glucuronidase activity (Tables 3 and 4 ), but these strains were not the same two strains that were stimulated by galacturonic acid.

Enzyme activities of spirochetes. Table 3 shows a summary of the distribution of enzyme activities in our collection of cultivated oral treponemes as detected by API ZYM tests. Catalase tests were negative for all of our strains of oral spirochetes. These results are consistent with previously published data $(8,10,18,29)$; however, a $\beta$-glucuronidase activity which we found to be characteristic for $T$. socranskii subsp. buccale does not appear to have been described for $T$. socranskii before. The heterogeneity of the novel isolates was more pronounced than is evident from Table 3 since the enzyme activity levels differed widely between strains, with scores ranging from 0 to 5 for $\alpha$-galactosidase and $\alpha$-fucosidase activities and from 0 to 3 for $\beta$-galactosidase and $\beta$-glucosidase activities; the levels of $\alpha$-glucosidase activity, which was present in all 26 new strains, ranged from 1 to 5 .

Antibiotics for selective isolation of spirochetes. Initially, a combination of rifampin and nalidixic acid was used to selectively suppress nonspirochetal growth from subgingival plaque samples. While this procedure resulted in isolation of many spirochetes, including phenotypically novel strain $\mathrm{BR}^{\mathrm{T}}$, the results obtained with numerous samples indicated that nalidixic acid was not an effective selecting agent (data not shown). To develop an improved selection scheme, we examined the antibiotic susceptibilities of representative strains of spirochetes by using a microtiter culture assay. Table 5 shows the levels of resistance of new isolate $\mathrm{BR}^{\mathrm{T}}$ and one strain each of $T$. denticola, $T$. pectinovorum, $T$. socranskii, and $T$. vincentii to cycloserine, fosfomycin, polymyxin $\mathrm{B}$, and rifampin; in addition, all five of these strains were found to be susceptible to erythromycin ( $1 \mathrm{mg} /$ liter), spectinomycin ( $5 \mathrm{mg} / \mathrm{liter}$ ), and vancomycin (5 mg/liter). When tested on subgingival plaque samples at the highest concentration compatible with the treponemes tested, polymyxin B (1 mg/liter; 7,900 U/liter) and cycloserine $(10 \mathrm{mg} / \mathrm{liter})$ had only minor effects on nonspirochetal cell counts in our media, either alone or in combination with $1 \mathrm{mg}$ of rifampin per liter (data not shown). Therefore, combinations of rifampin (1 mg/liter) and fosfomycin (100 or $1,000 \mathrm{mg} /$ liter) were subsequently used for spirochete isolation.

Phylogenetic analysis. Partial 16S rRNA sequences (length, 

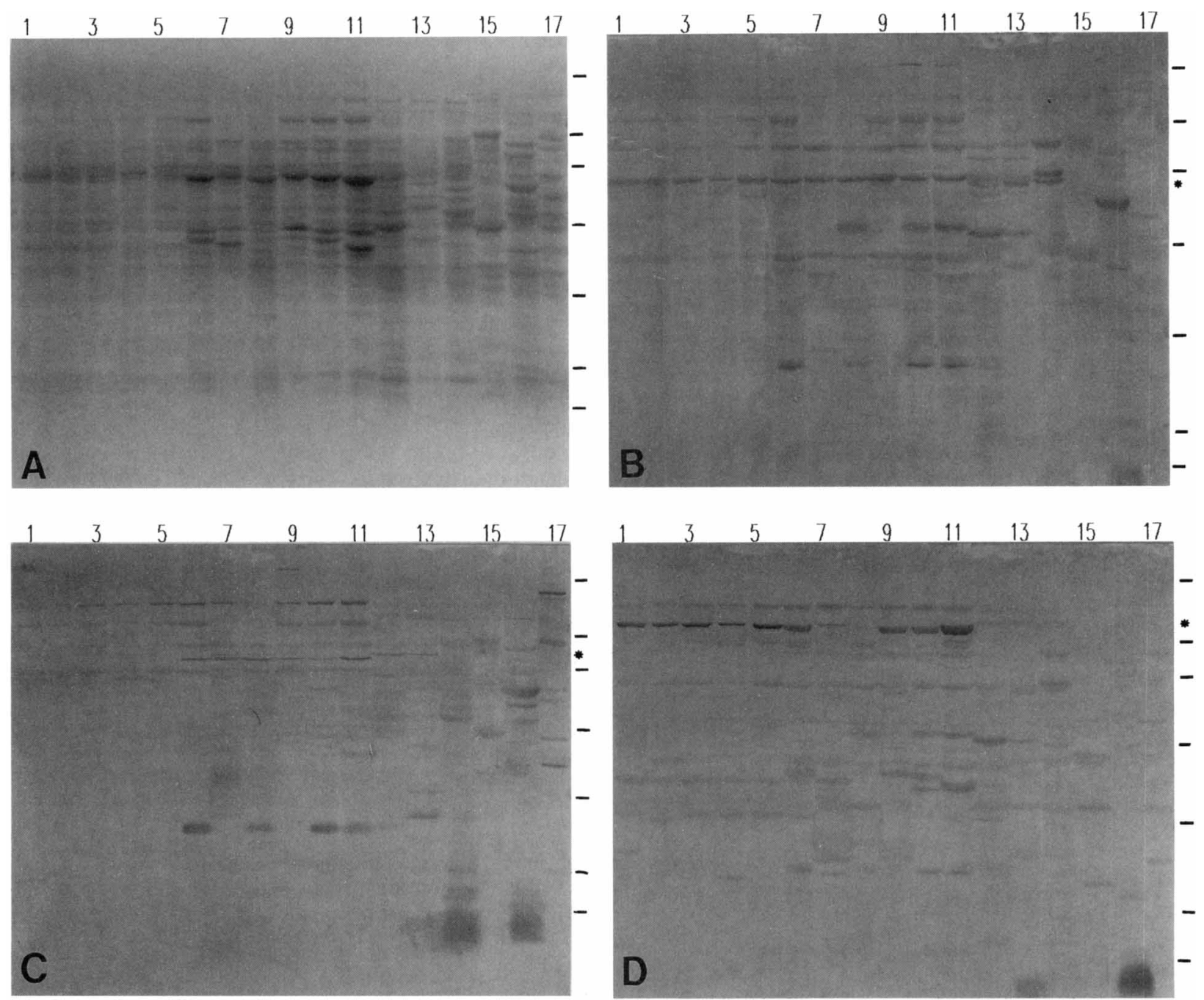

FIG. 2. Protein and antigen patterns of representative strains of cultivable oral spirochetes after SDS-PAGE on a 7.5 to $15 \%$ polyacrylamide gradient and electroblotting onto nitrocellulose. Lanes 1 through 11, novel isolates (lanes 1, HO2A; lanes 4, PNA1; lanes 11, BR ${ }^{\text {) }}$; lanes 12, T. socranskii subsp. socranskii ATCC $35536^{\mathrm{T}}$; lanes $13, T$. socranskii subsp. paredis ATCC $35535^{\mathrm{T}}$; lanes $14, T$. socranskii subsp. buccale ATCC $35534^{\mathrm{T}}$; lanes 15 , T. pectinovorum ATCC $33768^{\mathrm{T}}$; lanes 16 , T. denticola CD-1; lanes 17, T. vincentii LA-1 (= ATCC 35580). (A) Gel stained for protein. (B through D) Parallel blots immunostained with sera from periodontitis patients after the protein stain was removed. The serum used for panel $\mathrm{B}$ was from a patient from whom no spirochetes were isolated. Panel $\mathrm{C}$ shows the blot in panel $\mathrm{A}$ immunostained with the serum from the patient harboring strain $\mathrm{BR}^{\mathrm{T}}$ (lane 11). The blot in panel $\mathrm{D}$ was immunostained with the serum from the patient harboring the strains in lanes 5, 7, and 8. The positions of molecular weight markers (molecular weights [from top to bottom], 200,000, 97,000,69,000, 46,000, 30,000, 21,000, and $13,000)$ are indicated on the right. The asterisks indicate the positions of antigens specific for the novel isolates in panels $B$ and $D$ and the new strains with $\alpha$-glucosidase activity in panel $\mathbf{C}$.

about 350 to 500 bases) of 14 of the 26 new spirochete isolates, including strains $\mathrm{BR}^{\mathrm{T}}, \mathrm{HO} 2 \mathrm{~A}$, and PNA1, were determined for a phylogenetic classification analysis. A comparative sequence analysis revealed that there were only minor differences among the members of this phenotypically heterogeneous group and that they were closely related to all cluster 17 phylotypes except clone NZM3113 (6). The $16 S$ rRNA sequence of strain BR $^{\mathrm{T}}$ was identical to that of clone NZM3125. The overall levels of sequence similarity between strain $\mathrm{BR}^{\mathrm{T}} 16 \mathrm{~S}$ rRNA and the $16 \mathrm{~S}$ rRNAs of other cultivable oral treponemes ranged from 84 to 87.5\% (Table 6 and Fig. 3).

\section{DISCUSSION}

The experience of workers in many laboratories that all oral spirochetes isolated by the previously described methods belong to the four previously recognized oral Treponema species
TABLE 1. Growth of novel isolates depending on medium components

\begin{tabular}{|c|c|c|c|c|c|c|c|}
\hline \multirow{3}{*}{ Strain } & \multirow{3}{*}{ GlucNac } & \multicolumn{6}{|c|}{ Growth $^{a}$} \\
\hline & & \multicolumn{3}{|c|}{$-A s F$} & \multicolumn{3}{|c|}{$+\mathrm{AsF}$} \\
\hline & & YEM & DANP & $\begin{array}{c}\text { YEM + } \\
\text { DANP }\end{array}$ & YEM & DANP & $\begin{array}{c}\text { YEM + } \\
\text { DANP }\end{array}$ \\
\hline \multirow[t]{2}{*}{$\mathrm{BR}^{\mathrm{T}}$} & - & - & - & - & - & - & - \\
\hline & + & $(+)$ & - & - & + & $(+)$ & $(+)$ \\
\hline \multirow[t]{2}{*}{$\mathrm{HO} 2 \mathrm{~A}$} & - & + & - & + & + & - & + \\
\hline & + & + & - & + & + & + & + \\
\hline \multirow[t]{2}{*}{ PNA1 } & - & - & - & - & + & - & + \\
\hline & + & - & - & $(+)$ & + & - & + \\
\hline
\end{tabular}

${ }^{a}$ Growth was determined after 6 days of incubation in semisolid media with or without the medium components indicated. -, no growth; $(+)$, intermediate growth; + , good growth. 
TABLE 2. Growth of oral treponemes in response to different sugar additives

\begin{tabular}{|c|c|c|c|c|c|c|c|c|c|c|c|c|c|c|c|c|c|c|}
\hline \multirow[b]{2}{*}{ Taxon } & \multirow[b]{2}{*}{ 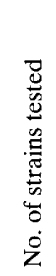 } & \multicolumn{17}{|c|}{ No. of strains that grew with the following sugar: } \\
\hline & & हूँ & 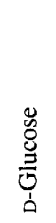 & 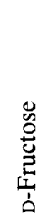 & 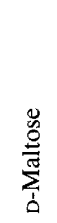 & 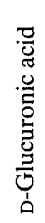 & 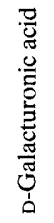 & 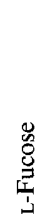 & 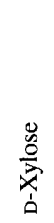 & 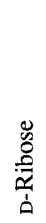 & 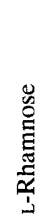 & 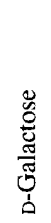 & 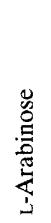 & 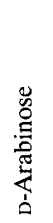 & 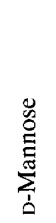 & $\begin{array}{l}\frac{0}{0} \\
\frac{0}{0} \\
\frac{0}{0} \\
\sum_{\dot{0}}^{0} \\
\dot{0}\end{array}$ & 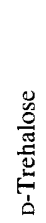 & 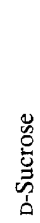 \\
\hline Novel isolates & $26^{a}$ & 3 & 12 & 12 & 23 & 2 & 2 & 24 & 9 & 23 & 19 & 11 & 11 & 20 & 14 & 7 & 22 & 17 \\
\hline T. denticola & 42 & 42 & $7^{b}$ & $-^{c}$ & $4^{b}$ & - & - & - & - & - & - & $6^{b}$ & - & - & $3^{b}$ & - & - & - \\
\hline T. pectinovorum & 4 & 0 & - & - & - & 4 & 4 & - & - & - & - & - & - & - & - & - & - & - \\
\hline T. socranskii subsp. buccale & 4 & 0 & - & 1 & 4 & 1 & - & - & - & 4 & 2 & - & 2 & - & - & - & 4 & 1 \\
\hline T. socranskii subsp. paredis & 4 & 0 & 2 & - & - & - & - & - & - & - & - & - & - & - & 2 & - & - & 3 \\
\hline T. socranskii subsp. socranskii & 13 & 0 & 2 & 8 & 12 & - & - & 1 & 12 & 10 & 8 & 4 & 11 & 12 & 5 & - & 6 & 5 \\
\hline T. vincentii & 4 & 4 & $1^{b}$ & - & $1^{b}$ & - & - & - & $1^{b}$ & - & - & $1^{b}$ & - & - & $1^{b}$ & - & - & - \\
\hline
\end{tabular}

${ }^{a}$ Strains BO2A, BO2B, BR ${ }^{\mathrm{T}}$, CRG3, GRA1, GRA2, GRA3, GRA4, GRB3A, GRB5, GRB6, GRB7, HO1, HO2A, HO2B, MH3B, PE1, PE2A, PE2B, PE2C, PE2D, PE2E, PNA1, PNA3, PNB1, and VF2D.

${ }^{b}$ Growth was enhanced compared with the control.

$c$, no effect on growth.

suggested that further progress in the cultivation of oral spirochetes would require new methods. Fastidious organisms are characterized not only by extensive auxotrophies for growth but also by marked sensitivity to growth inhibition. With these characteristics in mind, we previously developed a chemically defined and yet very nutritionally rich medium, OMIZ-W1 (37). All attempts to isolate oral spirochetes in OMIZ-W1 were unsuccessful, and so a medium enriched with carbohydrates, glutathione, AsF, and the chemically undefined extracts YEM and DANP was tried. In contrast to our laboratory strains, most of the clinical treponemal strains isolated in this way required at least one of these supplements. We are now investigating whether the complex additives play a direct nutritional role or act by neutralizing substances in the medium that are otherwise inhibitory.

During our studies with OMIZ-Pat, we have never been able to isolate spirochetes without antibiotic selection, even from plaque samples containing more than $60 \%$ spirochetes, as determined by dark-field microscopy. In contrast to reports claiming representative cultivation of all of the spirochetes present in plaque (26) or viable numbers that even exceed the microscopic counts (21), our treponemal isolates generally represented less than $1 \%$ of the organisms growing in OMIZ-Pat without antibiotics (data not shown), and so the success of isolating spirochetes did not directly correlate with spirochete levels in the plaque samples as determined by dark-field microscopy; indeed, samples that contained no more than $3 \%$ spirochetes have yielded growing treponemes, whereas other samples that contained up to $68 \%$ total spirochetes have failed to yield growing treponemes. The reasons for these results are not known, but it seems plausible that nonviability of some morphologically recognized treponemes, inefficient recovery of cultivable Treponema spp., and the presence in high proportions of still uncultivable spirochete species all contribute to the underrepresentation of spirochetes in the cultured plaque population. Similar discrepancies between microscopic and culture data have been reported in studies in which spirochetes were selectively grown in the absence of antibiotics by exploitation of their ability to migrate through Millipore membrane filters (20).

The use of antibiotic selection in attempts to isolate organisms with unknown physiological characteristics is obviously

TABLE 3. Numbers of oral treponeme strains that exhibit enzyme activities as detected by the API ZYM test

\begin{tabular}{|c|c|c|c|c|c|c|c|c|c|c|c|c|c|c|c|c|c|c|c|c|}
\hline \multirow[b]{2}{*}{ Taxon } & \multirow[b]{2}{*}{$\begin{array}{l}\text { No. of strains } \\
\text { tested }\end{array}$} & \multicolumn{19}{|c|}{ No. of strains that exhibit the following enzyme activity: } \\
\hline & & 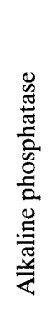 & 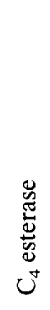 & 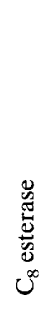 & 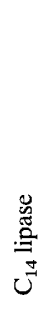 & 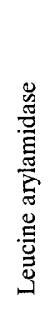 & 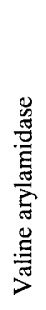 & 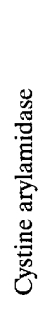 & $\stackrel{\Xi}{E}$ & 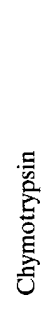 & 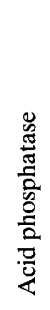 & 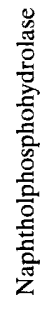 & 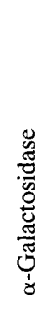 & 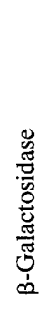 & 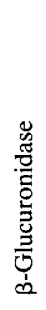 & 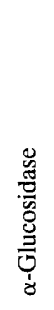 & 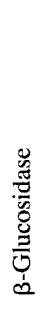 & 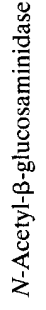 & 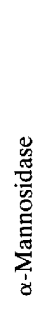 & 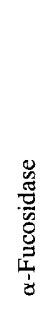 \\
\hline Novel isolates & 26 & 26 & 26 & 26 & 0 & 1 & 0 & 1 & 0 & 0 & 26 & 26 & 24 & 24 & 2 & $26^{a}$ & 23 & 0 & 0 & 18 \\
\hline T. denticola & 66 & 63 & 66 & 65 & 0 & 58 & 0 & 13 & 66 & 57 & 60 & 65 & 43 & 42 & 0 & 16 & 37 & 0 & 0 & 1 \\
\hline T. pectinovorum & 4 & 2 & 3 & 2 & 0 & 0 & 0 & 0 & 0 & 0 & 4 & 4 & 0 & 0 & 1 & 0 & 0 & 0 & 0 & 0 \\
\hline T. socranskii subsp. buccale & 5 & 5 & 5 & 4 & 1 & 0 & 0 & 0 & 0 & 1 & 5 & 5 & 0 & 0 & 5 & 0 & 0 & 0 & 0 & 0 \\
\hline T. socranskii subsp. paredis & 4 & 4 & 4 & 3 & 0 & 0 & 0 & 0 & 0 & 0 & 4 & 4 & 0 & 0 & 0 & 0 & 0 & 0 & 0 & 0 \\
\hline T. socranskii subsp. socranskii & 12 & 12 & 12 & 4 & 0 & 7 & 0 & 0 & 0 & 1 & 12 & 12 & 0 & 0 & 0 & 0 & 0 & 0 & 0 & 0 \\
\hline T. vincentii & 4 & 0 & 2 & 2 & 0 & 4 & 1 & 0 & 0 & 0 & 4 & 4 & 0 & 4 & 0 & 0 & 0 & 4 & 0 & 0 \\
\hline
\end{tabular}

${ }^{a}$ Boldface type indicates an enzyme activity that is distinctive for a taxon. 
TABLE 4. Levels of enzyme activities in oral treponemes as determined by the API ZYM test

\begin{tabular}{|c|c|c|c|c|c|c|c|c|c|c|c|c|c|c|c|c|c|c|c|c|}
\hline \multirow[b]{2}{*}{ Taxon } & \multirow[b]{2}{*}{ Strain } & \multicolumn{19}{|c|}{ Level of the following enzyme activity: } \\
\hline & & 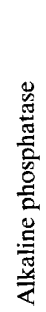 & 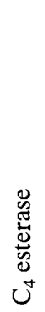 & 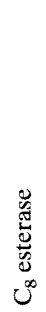 & 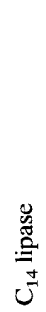 & 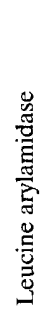 & 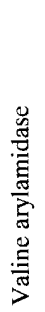 & 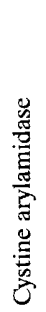 & 量 & 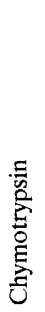 & 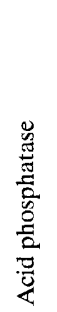 & 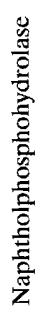 & 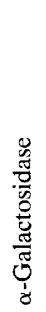 & 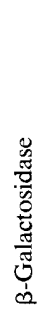 & 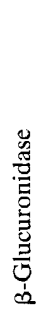 & 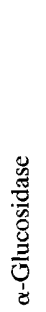 & 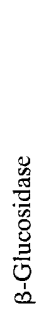 & 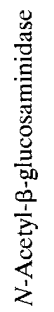 & 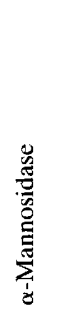 & 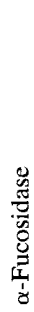 \\
\hline \multirow[t]{3}{*}{ Novel isolates } & $\mathrm{BR}^{\mathrm{T}}$ & 5 & 2 & 1 & 0 & 0 & 0 & 0 & 0 & 0 & 5 & 5 & 1 & 0 & 0 & 3 & 0 & 0 & 0 & 4 \\
\hline & $\mathrm{HO} 2 \mathrm{~A}$ & 5 & 2 & 1 & 0 & 0 & 0 & 0 & 0 & 0 & 5 & 3 & 1 & 1 & 4 & 3 & 1 & 0 & 0 & 0 \\
\hline & PNA1 & 3 & 2 & 2 & 0 & 0 & 0 & 0 & 0 & 0 & 5 & 1 & 1 & 3 & 0 & 3 & 1 & 0 & 0 & 0 \\
\hline \multirow[t]{2}{*}{ T. denticola } & $\mathrm{CD}-1$ & 3 & 3 & 3 & 0 & 2 & 0 & 0 & 5 & 2 & 3 & 3 & 2 & 0 & 0 & 0 & 0 & 0 & 0 & 0 \\
\hline & $51 \mathrm{~B} 2$ & 5 & 2 & 2 & 0 & 4 & 0 & 2 & 5 & 1 & 3 & 5 & 1 & 1 & 0 & 0 & 1 & 0 & 0 & 0 \\
\hline T. pectinovorum & ATCC $33768^{\mathrm{T}}$ & 0 & 2 & 2 & 0 & 0 & 0 & 0 & 0 & 0 & 2 & 1 & 0 & 0 & 0 & 0 & 0 & 0 & 0 & 0 \\
\hline T. socranskii subsp. buccale & ATCC $35534^{\mathrm{T}}$ & 5 & 3 & 1 & 0 & 0 & 0 & 0 & 0 & 0 & 5 & 4 & 0 & 0 & 5 & 0 & 0 & 0 & 0 & 0 \\
\hline T. socranskii subsp. paredis & ATCC $35535^{\mathrm{T}}$ & 2 & 3 & 1 & 0 & 0 & 0 & 0 & 0 & 0 & 5 & 1 & 0 & 0 & 0 & 0 & 0 & 0 & 0 & 0 \\
\hline T. socranskii subsp. socranskii & ATCC $35536^{\mathrm{T}}$ & 4 & 2 & 0 & 0 & 0 & 0 & 0 & 0 & 0 & 5 & 3 & 0 & 0 & 0 & 0 & 0 & 0 & 0 & 0 \\
\hline \multirow[t]{2}{*}{ T. vincentii } & LA-1 & 0 & 0 & 0 & 0 & 5 & 0 & 0 & 0 & 0 & 3 & 1 & 0 & 4 & 0 & 0 & 0 & 3 & 0 & 0 \\
\hline & Ritz A & 0 & 1 & 1 & 0 & 5 & 0 & 0 & 0 & 0 & 1 & 1 & 0 & 3 & 0 & 0 & 0 & 1 & 0 & 0 \\
\hline
\end{tabular}

problematic. From our perspective, any evidence of treponeme-inhibiting activity should caution against the use of the antibiotic that exhibits this activity. Conflicting data, possibly due to medium and strain differences, have been published regarding the susceptibility of oral treponemes to polymyxin and nalidixic acid $(4,9,30)$ (Table 5); in contrast, rifampin has been widely used since its first use with oral spirochetes by Leschine and Canale-Parola (15), and no inhibitory effects have been reported except for the susceptibility to a high dose $(10 \mathrm{mg} /$ liter) exhibited by two of the five treponemes which we tested. Spectinomycin resistance and rifampin susceptibility have been reported to be physiological characteristics that distinguish intestinal spirochetes from oral spirochetes (12). When the organisms were grown in our media, the difference in spectinomycin susceptibility could be confirmed; however, neither Serpulina jonesii ATCC 49776 nor Brachyspira aalborgii ATCC 43993 was inhibited by $10 \mathrm{mg}$ of rifampin per liter, nor were three strains of Borrelia burgdorferi (data not shown). Similarly, all of our oral and nonoral spirochetes were resistant to fosfomycin at a concentration of $100 \mathrm{mg} /$ liter, and most strains were resistant to even higher concentrations. This antibiotic, which has not been used previously for selection of oral spirochetes, was successfully used to isolate Lyme disease spirochetes $(16,23)$. The combined data for treponemal resistance and nontreponemal susceptibility in our media led us to use routinely $1 \mathrm{mg}$ of rifampin per liter plus $100 \mathrm{mg}$ of fosfomycin per liter for selective isolation of oral spirochetes.

While we do not have data on the efficiency of isolation, information about multiple isolates obtained from the same plaque sample supports the view that highly heterogeneous spirochete populations occur in plaque; not only have different species been isolated from the same sample, but isolates of the same species are often quite distinct. For example, only two of five novel strains isolated from one plaque sample were indistinguishable; all five strains exhibited $\alpha$-fucosidase activity, but the five strains produced four distinct protein and antigen patterns (Fig. 2, lanes 7 and 8) and had minor differences $(<2 \%)$ in their rRNA sequences (data not shown). The only novel strain isolated from a different pocket of the same patient lacked $\alpha$-fucosidase activity and differed antigenically (Fig. 2, lane 5).
On the basis of a phylogenetic characterization of 14 of the 26 new isolates and a comprehensive phenotypic comparison of all 26 isolates, we concluded that these small oral spirochetes belong to a single species. According to Stackebrandt and Goebel (28), a novel species should be created for organisms which exhibit less than $97 \%$ 16S rRNA sequence homology to previously described species. The data in the similarity matrix constructed for strain $\mathrm{BR}^{\mathrm{T}}$ and 12 other spirochetes (Table 6) clearly support the species status of the new group. The phylogenetic relationships of strain $\mathrm{BR}^{\mathrm{T}}$ and 25 previously described species of spirochetes are shown in Fig. 3. The level of 16S rRNA sequence homology between strain $\mathrm{BR}^{\mathrm{T}}$ and 13 novel isolates, including $\mathrm{HO} 2 \mathrm{~A}$ and PNA1, was found to be $98.5 \%$ on the basis of a comparison of 500 bases. This value should increase to more than $99 \%$ when the entire sequences are available, since most hypervariable sequences in the $16 \mathrm{~S}$ rRNAs of spirochetes are located in the region that we sequenced (25). The high level of sequence homology for our novel isolates and the low levels of sequence similarity between the novel isolates and other spirochetes therefore indicate that a new species should be described. Because of the characteristic $\alpha$-glucosidase activity and growth on maltose, we propose that the new species should be named Treponema maltophilum.

TABLE 5. Antibiotic resistance of cultured oral treponemes

\begin{tabular}{lcccc}
\hline \multirow{2}{*}{ Strain } & \multicolumn{4}{c}{$\begin{array}{c}\text { Highest concn (mg/iter) of the following } \\
\text { antibiotic that did not inhibit growth }\end{array}$} \\
\cline { 2 - 5 } & Rifampin & Polymyxin B & Cycloserine & Fosfomycin \\
\hline Novel isolate $\mathrm{BR}^{\mathrm{T}}$ & $>10$ & 1.25 & 100 & $>1,000$ \\
$T$. pectinovorum & $>10$ & 1.25 & 10 & $>1,000$ \\
$\quad$ ATCC 33768 & & & & \\
T. denticola CD-1 & 1 & 12.5 & 10 & $>1,000$ \\
T. socranskii & $>10$ & 12.5 & 100 & $>1,000$ \\
ATCC 35536 & & & & \\
T. vincentii LA-1 & 1 & 12.5 & $<10$ & 100 \\
\hline
\end{tabular}

${ }^{a}$ The following concentrations of antibiotics were tested: $0.1,1$, and $10 \mathrm{mg}$ of rifampin per liter; $1.25,12.5$, and $125 \mathrm{mg}$ of polymyxin $\mathrm{B}$ per liter; 10,100 , and $1,000 \mathrm{mg}$ of cycloserine per liter; and $1,10,100$, and $1,000 \mathrm{mg}$ of fosfomycin per liter. 
TABLE 6. 16S rRNA sequence similarity matrix for novel treponeme strain $\mathrm{BR}^{\mathrm{T}}$ and reference organisms

\begin{tabular}{|c|c|c|c|c|c|c|c|c|c|c|c|}
\hline \multirow[b]{2}{*}{ Taxon } & \multicolumn{11}{|c|}{$\%$ rRNA sequence similarity ${ }^{a}$} \\
\hline & 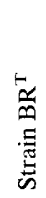 & 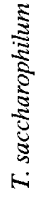 & 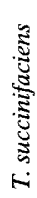 & 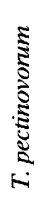 & $\frac{\tilde{3}}{3}$ & 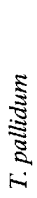 & 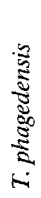 & 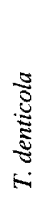 & 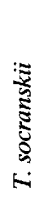 & 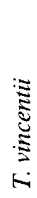 & 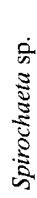 \\
\hline
\end{tabular}

T. saccharophilum 82.5

T. succinifaciens $\quad 80.982 .7$

T. pectinovorum 87.586 .283 .6

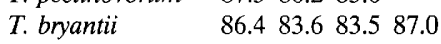

T. pallidum $\quad 84.582 .882 .684 .184 .2$

T. phagedensis $\quad 86.482 .980 .184 .684 .890 .4$

T. denticola $\quad 87.083 .781 .085 .485 .189 .492 .3$

$\begin{array}{lllllllll}\text { T. socranskii } \quad 84.0 & 81.3 & 83.9 & 84.9 & 84.0 & 82.9 & 83.8 & 84.2\end{array}$

T. vincentii $\quad 84.480 .4 \quad 77.983 .482 .3 \quad 85.790 .489 .180 .1$

$\begin{array}{llllllllllll}\text { Spirochaeta sp. } \quad 85.2 & 83.0 & 82.4 & 83.9 & 85.3 & 87.0 & 86.1 & 87.0 & 83.2 & 86.0\end{array}$

$\begin{array}{llllllllllll}\text { Treponema sp. } \quad 84.6 & 87.9 & 88.2 & 86.6 & 86.0 & 84.7 & 83.9 & 83.8 & 85.1 & 81.7 & 84.9\end{array}$ strain CA

${ }^{a}$ Determined by a sequence pair comparison. For $T$. vincentii a 900-base partial sequence was compared.

Creation of subspecies based only on phenotypic differences and 16S rRNA sequence data does not seem to be warranted. Creation of subspecies will depend on the results of a DNADNA hybridization analysis, which is accepted as the most sensitive method for determining very close phylogenetic relationships, such as those that occur within species (28). Type strain BR and reference strains HO2A and PNA1 represent the wide phenotypic spectrum of this phylogenetically homogeneous group.

Description of Treponema maltophilum sp. nov. Treponema maltophilum (mal.to'phil.um. M.L. n. maltosum, maltose; Gr. adj. philos, loving, friendly to; M.L. adj. maltophilum, maltose loving) is an obligately anaerobic, helically coiled, motile treponeme isolated from human subgingival plaque. The cells are approximately $5 \mu \mathrm{m}$ long and $0.2 \mu \mathrm{m}$ wide and have a wavelength of $0.7 \mu \mathrm{m}$ and an amplitude of $0.3 \mu \mathrm{m}$. They have two periplasmic flagella; one flagellum originates at each cell end, and the flagella overlap in the central region of the cell. In low-viscosity liquid media cellular rotation produces standing waves with amplitudes of up to $2 \mu \mathrm{m}$, but this results in no directional motility. Translational movement, however, occurs in higher-viscosity media or when cells creep along a surface. Cells can be stored frozen (in liquid nitrogen or in a mechanical freezer) in OMIZ-Pat supplemented with 10 to $20 \%$ glycerol.

Colonies. When streaked onto OMIZ-Pat agarose, T. maltophilum forms within 5 days off-white diffuse subsurface colonies that are up to $3 \mathrm{~mm}$ in diameter.

Cultural characteristics. T. maltophilum does not grow in chemically defined OMIZ-W1 and requires the addition of yeast extract and/or Neopeptone (or fractions thereof). Growth of most strains is strictly dependent on the presence of GlucNac and at least one additional sugar. The most commonly used second sugars are D-arabinose, L-fucose, D-maltose, L-rhamnose, D-ribose, D-sucrose, and D-trehalose, while D-glucose may be totally ineffective. Some strains without $\alpha$-fucosidase activity may not depend on GlucNac. Growth is not influenced by D-cellobiose, D-fucose, D-lactose, D-mannitol, L-sorbose, and Lxylose. FBS at concentrations as low as $0.1 \%$ (vol/ $/ \mathrm{vol}$ ) prevents growth in OMIZ-Pat. Catalase is not produced. As determined with API ZYM strips, all strains exhibit alkaline phosphatase, acid phosphatase, naphtholphosphohydrolase, $\mathrm{C}_{4}$ esterase, $\mathrm{C}_{8}$ esterase, and $\alpha$-glucosidase activities, and most strains are also positive for $\alpha$ - and $\beta$-galactosidase, $\beta$-glucosidase, and $\alpha$-fucosidase activities. Within the species there are prominent differences in $\alpha$-fucosidase and $\beta$-glucuronidase activities. Strains with $\beta$-glucuronidase activity are able to grow on glucuronic acid. Immunoblotting with patient sera reveals an antigen that can be detected only in strains with $\alpha$-fucosidase activity. All strains are resistant to $1 \mathrm{mg}$ of rifampin per liter and $100 \mathrm{mg}$ of fosfomycin per liter.

Type and reference strains. Strain BR is the type strain of T. maltophilum. This strain exhibits $\alpha$-fucosidase activity but not $\beta$-glucuronidase activity. Reference strains that differ markedly phenotypically from the type strain are strain $\mathrm{HO} 2 \mathrm{~A}$, which exhibits $\beta$-glucuronidase activity but not $\alpha$-fucosidase activity, and strain PNA1, which exhibits neither $\alpha$-fucosidase activity nor $\beta$-glucuronidase activity. Strains $\mathrm{BR}^{\mathrm{T}}, \mathrm{HO} 2 \mathrm{~A}$, and PNA1 have been deposited in the American Type Culture Collection, Rockville, Md., as strains ATCC 51939 ${ }^{\dagger}$, ATCC 51940, and ATCC 51941, respectively.

Habitat. T. maltophilum has been detected only in subgingival plaque samples of patients with periodontal disease.

Distinguishing characteristics. T. maltophilum is a species that is genetically distinct from previously described treponemes as determined by a phylogenetic comparison of $16 \mathrm{~S}$ rRNA sequences. As determined by SDS-PAGE, its protein and antigen patterns can be easily distinguished from those of other cultivable treponemes, although more conventional criteria can also be used to distinguish it from the four previously

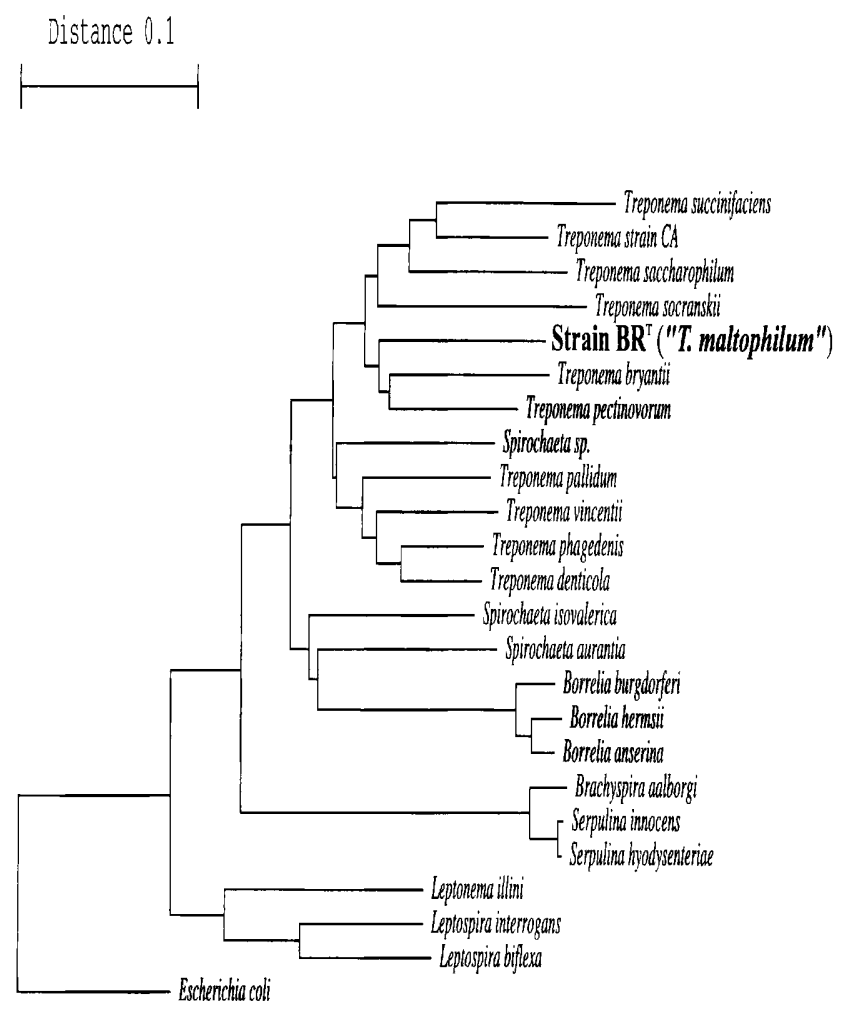

FIG. 3. Phylogenetic tree showing the relationships of novel strain $\mathbf{B R}^{\mathrm{T}}$ to other spirochetes based on a comparison of 16S rRNA sequences (about 900 bases). E. coli was used as the outgroup. Scale bar $=10 \%$ difference in nucleotide sequences as determined by measuring the lengths of the horizontal lines connecting two sequences. 
characterized oral spirochetes. Size, morphology, enzyme activities, and growth characteristics clearly distinguish $T$. maltophilum from $T$. vincentii. $T$. maltophilum also differs from the asaccharolytic organism $T$. denticola by its lack of trypsin activity and from $T$. pectinovorum by its lack of a requirement for either glucuronic acid or galacturonic acid. Differentiation from $T$. socranskii is based on the wide spectrum of glycosidases produced by $T$. maltophilum, as well as on the spectrum of carbohydrates used. $\alpha$-Glucosidase activity is characteristic of T. maltophilum and has never been observed in T. socranskii, although many strains of this species can ferment maltose. A distinctive phenotypic trait found in all strains of all subspecies of $T$. socranskii but not in $T$. maltophilum or any other oral spirochete is the formation of intensely yellow colonies (or cell pellets after growth in liquid medium) in OMIZ-Pat agarose (data not shown). This includes an oral Treponema strain of uncertain classification described by Mink (19) and obtained from the American Type Culture Collection as strain ATCC 43242, which we identified by protein, antigen, API ZYM, and sugar utilization analyses as $T$. socranskii subsp. socranskii (data not shown). Yellow colonies have been described by Umeda et al. (30), but they were not identified as T. socranskii colonies. Furthermore, live cells of $T$. socranskii can be recognized microscopically, since cells that are rotating around their axes have both cell tips markedly deflected, which gives the appearance of propellers on both ends of a straight helix (data not shown).

\section{ACKNOWLEDGMENTS}

We thank C. Weiss and V. Zängerle for expert technical assistance and S. Shapiro for a critical review of the manuscript.

This work was supported in part by a grant from the Deutsches Bundesministerium für Bildung und Forschung.

\section{REFERENCES}

1. Bickar, D., and P. D. Reid. 1992. A high-affinity protein stain for Western blots, tissue prints, and electrophoretic gels. Anal. Biochem. 203:109-115.

2. Brenner, A., and R. W. Horne. 1959. A negative staining method for the high resolution electron microscopy of viruses. Biochim. Biophys. Acta 34:103110

3. Casanova, J.-L., C. Pannetier, C. Jaulin, and P. Kourilsky. 1990. Optimal conditions for directly sequencing double-stranded PCR products with sequenase. Nucleic Acids Res. 18:4028.

4. Chan, E. C. S., R. Siboo, L. Z. G. Touyz, Y.-S. Qiu, and A. A. Klitorinos. 1993. A successful method for quantifying viable oral anaerobic spirochetes. Oral Microbiol. Immunol. 8:80-83.

5. Choi, B. K., and U. B. Göbel. Unpublished data

6. Choi, B. K., B. J. Paster, F. E. Dewhirst, and U. B. Göbel. 1994. Diversity of cultivable and uncultivable oral spirochetes from a patient with severe destructive periodontitis. Infect. Immun. 62:1889-1895.

7. Dewhirst, F. E. Personal communication.

8. Fiehn, N.-E. 1987. Biochemical characterization of nine oral small-sized spirochete strains containing one endoflagellum from each cell-end. APMIS 95:309-314.

9. Fiehn, N.-E., and A. Frandsen. 1984. Evaluation of serum-containing substrates for cultivation of oral spirochetes. J. Periodontal Res. 19:61-66.

10. Fukumoto, Y., K. Okuda, T. Kato, K. Ohta, and I. Takazoe. 1987. Taxonomic study of spirochetes isolated from human periodontal lesions. Oral Microbiol. Immunol. 2:82-87.

11. Holt, S. C., and T. E. Bramanti. 1991. Factors in virulence expression and their role in periodontal disease pathogenesis. Crit. Rev. Oral Biol. Med. 2 177-281.

12. Jones, M. J., J. N. Miller, and W. L. George. 1986. Microbiological and biochemical characterization of spirochetes from the feces of homosexual males. J. Clin. Microbiol. 24:1071-1074.

13. Jukes, T. H., and C. R. Cantor. 1969. Evolution of protein molecules, p. 21-132. In H. N. Munro (ed.), Mammalian protein metabolism. Academic Press, New York

14. Lane, D., B. Pace, G. J. Olsen, D. A. Stahl, M. L. Sogin, and N. R. Pace. 1985
Rapid determination of $16 \mathrm{~S}$ ribosomal RNA sequences for phylogenetic analysis. Proc. Natl. Acad. Sci. USA 82:6955-6959.

15. Leschine, S. B., and E. Canale-Parola. 1980. Rifampicin as a selective agent for isolation of oral spirochetes. J. Clin. Microbiol. 12:792-795.

16. Leuba-Garcia, S., M. D. Kramer, R. Wallich, and L. Gern. 1994. Characterization of Borrelia burgdorferi isolated from different organs of Ixodes ricinus ticks collected in nature. Zentralbl. Bakteriol. Parasitenkd. Infektionskr. Hyg. Abt. 1 Orig. 280:468-475.

17. Loesche, W. J., R. N. Hockett, and S. A. Syed. 1972. The predominant cultivable flora of tooth surface plaque removed from institutionalized subjects. Arch. Oral Biol. 17:1311-1325.

18. Mikx, F. H. M. 1991. Comparison of peptidase, glycosidase and esterase activities of oral and non-oral Treponema species. J. Gen. Microbiol. 137:6368.

19. Mink, R. W. 1984. Isolation of a new saccharolytic oral spirochete from diseased periodontal pocket, abstr. C160, p. 263. In Abstracts of the 84th Annual Meeting of the American Society for Microbiology 1984. American Society for Microbiology, Washington, D.C.

20. Moore, W. E. C., L. V. Hóldeman, R. M. Smibert, E. P. Cato, J. A. Burmeister, K. G. Palcanis, and R. R. Ranney. 1984. Bacteriology of experimental gingivitis in children. Infect. Immun. 46:1-6.

21. Moore, W. E. C., L. V. Holdeman, R. M. Smibert, D. E. Hash, J. A. Burmeister, and R. R. Ranney. 1982. Bacteriology of severe periodontitis in young adult humans. Infect. Immun. 38:1137-1148.

22. Moore, W. E. C., L. H. Moore, R. R. Ranney, R. M. Smibert, J. A. Burmeister, and H. A. Schenkein. 1991. The microflora of periodontal sites showing active destructive progression. J. Clin. Periodontol. 18:729-739.

23. Morshed, M. G., H. Konishi, T. Nishimura, and T. Nakazawa. 1993. Evaluation of agents for use in medium for selective isolation of Lyme disease and relapsing fever Borrelia species. Eur. J. Clin. Microbiol. Infect. Dis. 12: $512-518$.

24. Offenbacher, S., J. G. Collins, and R. R. Arnold. 1993. New clinical diagnostic strategies based on pathogenesis of disease. J. Periodontal Res. 28:523535.

25. Paster, B. J., F. E. Dewhirst, W. G. Weisburg, L. A. Tordoff, G. J. Fraser, R. B. Hespell, T. B. Stanton, L. Zablen, L. Mandelco, and C. R. Woese. 1991. Phylogenetic analysis of the spirochetes. J. Bacteriol. 173:6101-6109.

26. Qiu, Y.-S., A. Klitorinos, M. D. Rahal, R. Siboo, and E. C. S. Chan. 1994 Enumeration of viable oral spirochetes from periodontal pockets. Oral Microbiol. Immunol. 9:301-304.

27. Saitou, N., and M. Nei. 1987. The neighbor-joining method: a new method for reconstructing phylogenetic trees. Mol. Biol. Evol. 4:406-425.

28. Stackebrandt, E., and B. M. Goebel. 1994. Taxonomic note: a place for DNA-DNA reassociation and 16S rRNA sequence analysis in the present species definition in bacteriology. Int. J. Syst. Bacteriol. 44:846-849.

29. Syed, S. A., S. L. Salvador, and W. J. Loesche. 1988. Enzyme profiles of oral spirochetes in RapID-ANA system. J. Clin. Microbiol. 26:2226-2228.

30. Umeda, M., I. Ishikawa, Y. Benno, and T. Mitsuoka. 1990. Improved detection of oral spirochetes with an anaerobic culture method. Oral Microbiol. Immunol. 5:90-94.

31. Van De Peer, Y., and R. De Wachter. 1993. TREECON: a software package for the construction and drawing of evolutionary trees. Comput. Applic. Biosci. 9:177-182.

32. Weisburg, W. G., S. M. Barns, D. A. Pelletier, and D. J. Lane. 1991. 16S ribosomal DNA amplification for phylogenetic study. J. Bacteriol. 173:697703.

33. West, S., J. Schröder, and W. Kunz. 1990. A multiple-staining procedure for the detection of different DNA fragments on a single blot. Anal. Biochem. 190:254-258

34. Westergaard, J., and N.-E. Fiehn. 1987. Morphological distribution of spirochetes in subgingival plaque from advanced marginal periodontitis in humans. APMIS 95:49-55.

35. Wyss, C. 1989 . Campylobacter-Wolinella group organisms are the only oral bacteria that form arylsulfatase-active colonies on a synthetic indicator medium. Infect. Immun. 57:1380-1383.

36. Wyss, C. 1989. Dependence of proliferation of Bacteroides forsythus on exogenous $\mathrm{N}$-acetylmuramic acid. Infect. Immun. 57:1757-1759.

37. Wyss, C. 1992. Growth of Porphyromonas gingivalis, Treponema denticola, $T$. pectinovorum, $T$. socranskii, and $T$. vincentii in a chemically defined medium. J. Clin. Microbiol. 30:2225-2229.

38. Wyss, C. 1993. Aspartame as a source of essential phenylalanine for the growth of oral anaerobes. FEMS Microbiol. Lett. 108:255-258.

39. Wyss, C. 1995 . Sticky, a novel phenotype of Campylobacter rectus. Microb. Ecol. Health Dis. 8:175-179.

40. Wyss, C., P. Hunziker, and S. Klauser. 1993. Support of peptide dependent growth of Bacteroides forsythus by synthetic fragments of haemoglobin or fetuin. Arch. Oral Biol. 38:979-984. 